\title{
Right hippocampal contribution to visual memory: a presurgical and postsurgical study in patients with temporal lobe epilepsy
}

\author{
U Gleißner, C Helmstaedter, C E Elger
}

\begin{abstract}
Objectives-Existing speculations regarding the cognitive functions of the right hippocampus suggest a special involvement in non-verbal memory functions. However, all supporting evidence in epileptic patients came from postoperative studies that did not consider the preoperative functioning level. The present study investigates in patients with right temporal lobe epilepsy (RTLE), both preoperatively and postoperatively, the role of the right hippocampus for visual memory, visuoconstruction, and spatial visualisation.

Methods-Two groups of patients with RTLE with and without right hippocampal damage $\left(\mathrm{HC}^{+} / \mathrm{n}=15 v \mathrm{HC}^{-} / \mathrm{n}=13\right)$ were set up retrospectively thereby taking into account the surgical treatment (selective amygdalohippocampectomy $v$ temporal resections sparing the hippocampus), the postoperative pathological findings (hippocampal sclerosis in the $\mathrm{HC}^{+}$group) and the seizure outcome (all seizure free). The two groups were compared with regard to their neuropsychological performance preoperatively and 3 months after temporal lobe surgery.
\end{abstract}

Results-The preoperative results indicated impaired visual memory performance only for the $\mathrm{HC}^{+}$patients, whereas the $\mathrm{HC}^{-}$patients exhibited no deficiencies in any of the assessed functions. Postoperatively, both groups exhibited partially improved performances. The type of surgical treatment had no different effects on the postoperative performance changes.

Conclusions-The results suggest that hippocampal damage is the major cause of non-verbal memory deficiencies in preoperative patients with right temporal lobe epilepsy. Hence the results support and extend findings from previous postoperative studies. Right hippocampal resections did not increase the preoperative visual memory deficiencies, thus supporting the view that right temporal lobe resection in general bears only small risks for cognitve deteriorations.

(F Neurol Neurosurg Psychiatry 1998;65:665-669)

Keywords: right temporal lobe epilepsy, hippocampus, visual memory

Disorders of material specific learning and memory have long been associated with temporal lobe dysfunctions in focal epilepsy.
Whereas the left language dominant temporal lobe is consistently associated with memory impairments for verbal material, the right nondominant temporal lobe seems important for learning and memory of material that is difficult to verbalise such as unfamiliar faces or visually presented abstract designs (for a review see Milner ${ }^{1}$ ). Several studies in resected as well as in non-surgical patients confirm these material specific memory functions of the right temporal lobe. ${ }^{2-5}$ The deficiencies in visual memory in patients with right temporal lobe epilepsy typically involve learning performance or retention/recognition after a delay whereas short aspects of visual memory, such as immediate recall or memory span, often seem unimpaired. ${ }^{36}$ Cognitive changes after right temporal lobe surgery have not often been documented and determinants of the postoperative cognitive development are not yet clear. In general, right temporal lobe surgery seems to bear only small risks for additional cognitive deficiencies or exaggerations of preoperative impairments ${ }^{7}$; however, there are also studies reporting moderate cognitive declines after surgery. ${ }^{9}$

Recent research aims to find a functional differentiation within the temporal lobes. For the left temporal lobe there are the first hints that mesial and neocortical temporal structures may subserve different aspects of memory with data acquisitions or working memory being mediated by neocortical temporal structures and long term consolidation and retrieval being mediated by temporomesial structures. ${ }^{10}{ }^{11}$ For the right temporal lobe there is some evidence that damage specifically in temporomesial structures may be the cause of impairments in non-verbal memory functions. Milner ${ }^{12}$ found deficiencies in a visually guided maze learning task after right temporal lobectomy only when the operation included the hippocampal structures. Jones-Gotman et al reported in several studies relations between the severity of memory impairments and the extent of hippocampal, but not of neocortical, excisions in the right temporal lobe. ${ }^{13-15}$ However, all this evidence came from postoperative studies and did not consider the preoperative functioning level.

The goal of the present study was to evaluate the hippocampal contribution to visual memory by comparing neuropsychological performances of patients with RTLE (right temporal lobe epilepsy) with and without hippocampal damage preoperatively and postoperatively. In particular, we checked the ques-
Received 14 November 1997 30 April 1998

Accepted 29 May 1998 
Table 1 Patient characteristics

\begin{tabular}{|c|c|c|c|}
\hline & $\begin{array}{l}H C^{+} \text {group }(n=15) \\
\text { Mean }(S D)\end{array}$ & $\begin{array}{l}\text { HC group }(n=13) \\
\text { Mean }(S D)\end{array}$ & Significance \\
\hline Age (y) & $32.6(6.8)$ & $27.1(11.3)$ & $F=2.5 \mathrm{NS}$ \\
\hline Onset of epilepsy (y) & $11.5(5.4)$ & $14.7(9.4)$ & $F=0.5 \mathrm{NS}$ \\
\hline Duration of epilepsy (y) & $21.1(8.6)$ & $13.8(10.4)$ & $F=4.1 \mathrm{NS}$ \\
\hline Preoperative IQ & $101.6(10.6)$ & 109 (15) & $F=2.5 \mathrm{NS}$ \\
\hline \multicolumn{4}{|c|}{ Psychomotor speed (standard scores): } \\
\hline Preoperatively & $99.4(16.1)$ & $101.6(12.5)$ & $F=0.16 \mathrm{NS}$ \\
\hline Postoperatively & $107.7(14.4)$ & $113.9(9.3)$ & $F=1.7$ NS \\
\hline Male/female & $5 / 10$ & $7 / 6$ & $\chi^{2}=1.2 \mathrm{NS}$ \\
\hline \multicolumn{4}{|l|}{ Hand dominance: } \\
\hline Right/left/ambidextrous & $12 / 3 / 0$ & $10 / 1 / 2$ & $\chi^{2}=3.1 \mathrm{NS}$ \\
\hline
\end{tabular}

tions, whether already preoperatively a prominent association of non-verbal memory deficiencies with hippocampal damage can be found and whether right temporal lobe resections including or not including the hippocampus differentially affect the cognitive outcome. A functional differentiation of various structures within the right temporal lobe could improve the preoperative neuropsychological localisational diagnostics and could help to make predictions of the postoperative cognitive outcome in individual patients.

\section{Methods}

PATIENTS

Two groups of patients with RTLE were retrospectively set up: one group with damage restricted to the hippocampal structure $\left(\mathrm{HC}^{+}\right.$ group) and the other group with damage not involving the hippocampal structure ( $\mathrm{HC}^{-}$ group). Selection criteria for the $\mathrm{HC}^{+}$group were: selective amgydala-hippocampectomy as the surgical treatment, evidence of an ammons horn sclerosis in the histopathological finding, seizure free after surgery. Selection criteria for the $\mathrm{HC}^{-}$group were: no evidence of an ammons horn sclerosis or atrophy in the preoperative high resolution MRI, surgical treatment completely spared the hippocampal structure, seizure free after surgery. Because the most temporal lobe resections include the hippocampus, we found on the whole only 13 patients in our clinical population who fulfilled the $\mathrm{HC}^{-}{ }^{-}$riteria, but 47 patients who fulfilled the $\mathrm{HC}^{+}$criteria. To optimise the conditions for the statistical analyses we equalised the sample sizes by selecting out of the $47 \mathrm{HC}^{+}$patients a group of 15 patients who largely matched the $\mathrm{HC}^{-}$patients with respect to the age at surgery, the onset of epilepsy, and the sex ratio.

Surgical treatment in the $\mathrm{HC}^{-}$patients consisted of nine lesionectomies ( $+1 \mathrm{~cm}$ margins), three lobectomies, and one tailored resection. Specific sites of resections varied widely. Pathological findings in the $\mathrm{HC}^{-}$patients were "benign tumours" in 10 patients, "malign tumour" (astrocytoma WHO II) in one patient, and "other lesions" in two patients.

Surgical procedures had been preoperatively dictated by MRI findings and electrophysiological interictal and ictal recordings with surface and sphenoidal electrodes in all patients and intracranial recordings in nine patients (depth electrodes+subdural strips in seven patients, subdural strips in two patients).

Groups did not differ in clinical characteristics (table 1). Preoperative global intellectual functioning, estimated by a vocabulary test, ${ }^{16}$ as well as preoperative and postoperative psychomotor speed, assessed with a letter cancellation test, ${ }^{17}$ were average in both groups and there were no significant group differences. Hand dominance assessed with the Oldfield inventory ${ }^{18}$ indicated non-right hand dominance in six patients. These patients underwent preoperative intracarotid amytal tests, which indicated left hemispheric language dominance despite the atypical hand dominance.

NEUROPSYCHOLOGICAL MEASURES

We evaluated visual memory using a task which has already been proved to be sensitive to right temporal dysfunctions. ${ }^{19}$ Furthermore, we assessed visuospatial and visuoconstructional performances as right hemispheric cognitive functions, which are assumed not to specifically tap the temporal lobe.

Visual memory was assessed by the DCS-R, a revised version of the $\mathrm{DCS}^{20}$ (for detailed description see Helmstaedter et $a l^{19}{ }^{21}$ ). A parallel form was used in the postoperative examination. The DCS-R requires repeated learning of nine abstract designs (figure A) in six consecutive trials. The designs are presented consecutively on separate cards at a rate of 2 seconds per item. After each presentation the patient has to reconstruct as many items as possible using five wooden sticks of equal length. Patients are instructed to keep the design and the spatial arrangement identical with the target item. If the reproduction of the design is correct but its spatial positioning is mirrored or at least $30^{\circ}$ rotated, it is scored as a "rotation". If the reproduction of the design is incorrect, it is scored as a "false positive". After 30 minutes of a verbal distractor task, recognition of the original figures out of 21 distractors (nine rotated original designs and 12 similar designs) is required.

The evaluated indices were learning capacity (sum of correct reproductions in trials 1 to 6), rotations (sum of rotated designs in trials 1 to 6 ), failures (sum of false positives in trials 1 to 6 ), and recognition (correctly recognised items-falsely recognised distractors).

Visuoconstructive abilities were assessed using a German adaptation of the block design subtest of the WAIS. ${ }^{22}$ The evaluated variable was the age related total $T$ value.

Spatial visualisation was assessed using subtest 8 of the Leistungs-Prüf system. ${ }^{23}$ It requires matching of indicated positions in two dimensionally and three dimensionally drawn figures (figure B). The evaluated variable was the age related $\mathrm{c}$ value.

To illustrate results in comparison with healthy subjects and to facilitate the comparison of the different test performances, all results were uniformly transformed into standardised values (mean 100 (SD 10)) (for formula see f.i. in chapter 12 , Lienert ${ }^{24}$ ). Visual memory variables were standardised using values of a sample of 102 healthy controls (mean (SD) learning capacity 34.7 (9.6); rotations 2.4 (3.4); failures 6 (5.1); recognition 7 (1.9)) who corresponded to the patient groups for age (mean 29.8 (SD 8.8)) years and sex 

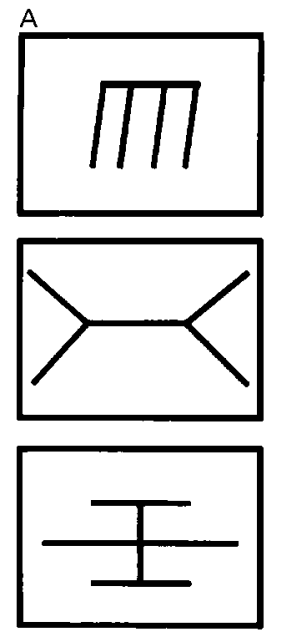

(A) These nine figures constitute the material in the non-verbal memory test DCS-R. (B) Example of the spatial visualisation task: each number in the two dimensional drawing on the left has a corresponding letter in the figure on the right, the identity of the number or letter has to be indicated by the patient.

ratio ( 50 males, 52 females). For the the block design test and the spatial visualisation task age related $T$ and $c$ values, provided in the test manuals, were transformed into standardised scores using the formulas: standard score $=100+10((\mathrm{~T}-50): 10)$ and standard score $=100+10((c-5): 2) .^{24}$

STATISTICAL ANALYSIS

Firstly, results are described in comparison with healthy controls. Secondly, preoperative group differences are evaluated by means of a multivariate analysis of variance (MANOVA) and subsequent unvariate analyses of variance (ANOVAs). Thirdly, postoperative data are evaluated with respect to the significance of performance changes (paired $t$ tests) and to significant group differences in the postoperative development (analyses of covariance incorporating the preoperative scores as covariates (ANCOVAs))

The statistical analyses were performed using the statistical package SPSS 6.0 for Windows.

\section{Results}

COMPARISON WITH HEALTHY CONTROLS

Preoperative and postoperative results are listed in table 2 . The representation in standardised scores allows a comparison with the performance of healthy controls. The $\mathrm{HC}^{+}$ patients scored preoperatively below average (standardised scores $<90$ ) in all variables of the visual memory task with learning capacity

Table 2 Preoperative and postoperative neuropsychological results

\begin{tabular}{llllll}
\hline & \multicolumn{2}{l}{ Preoperative $^{*}$} & & \multicolumn{2}{l}{ Postoperative $^{*}$} \\
\cline { 2 - 3 } \cline { 5 - 6 } Test index & $\begin{array}{l}\text { HC Mean } \\
(S D)\end{array}$ & $\begin{array}{l}\text { HC Mean } \\
(S D)\end{array}$ & & $\begin{array}{l}\text { HC Mean } \\
(S D)\end{array}$ & $\begin{array}{l}\text { HC Mean } \\
(S D)\end{array}$ \\
\hline Visual memory: & & & & & \\
$\quad$ Learning capacity & $80.2(10.3)$ & $96.6(10.4)$ & & $83.2(12.6)$ & $96.3(11.5)$ \\
$\quad$ Recognition & $86.7(12.7)$ & $97.0(13.2)$ & & $88.7(18.1)$ & $102.8(9.3)$ \\
$\quad$ Failures & $87.3(12.2)$ & $102.3(7.9)$ & & $94.5(12.2)$ & $101.5(7.6)$ \\
$\quad$ Rotations & $89.4(10.9)$ & $100.5(6.2)$ & & $98.4(9.7)$ & $100.5(7.9)$ \\
Spatial visualisation & $98.7(7.9)$ & $105.8(6.7)$ & & $100.9(8.5)$ & $106.9(8.3)$ \\
Visuoconstruction & $96.3(10.7)$ & $108.2(7.1)$ & & $101.3(11.9)$ & $112.8(8.9)$ \\
\hline
\end{tabular}

$\star$ Preoperative and postoperative neuropsychological results of patients with $\left(\mathrm{HC}^{+}\right)$and without $\left(\mathrm{HC}^{-}\right)$hippocampal damage (standardised scores).
B
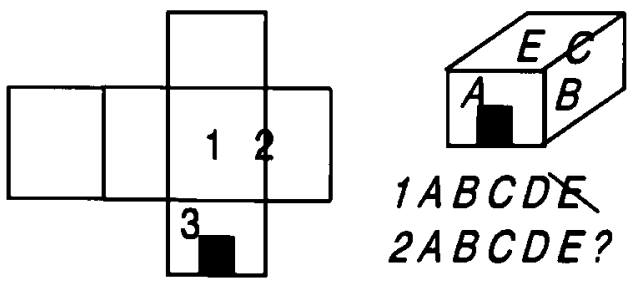

$1 A B C D K$ $2 A B C D E$ ? $3 A B C D E$ ? being the prominent impairment. Postoperatively, they showed impaired performance only in two indices of visual memory (learning capacity, recognition). Mean performances of the $\mathrm{HC}^{-}$patients were preoperatively and postoperatively completely within the normal range (mean $\pm 1 \mathrm{SD})$.

\section{PREOPERATIVE ANALYSES}

Preoperative group differences were first evaluated by a multivariate analysis, as the visual memory indices, except the rotational errors, were significantly correlated (Pearson correlations: $r$ between 0.4 and 0.76 with $\mathrm{p}<0.05)$. The MANOVA with surgical treatment $\left(\mathrm{HC}^{+} / \mathrm{HC}^{-}\right)$as the group factor and the neuropsychological indices as dependent variables yielded a significant overall group effect (Hotellings $\mathrm{T}=0.85$, exact $F=3.4, \mathrm{p}=0.002$ ), thus indicating that there were overall differences in the preoperative performance of the $\mathrm{HC}^{+}$and the $\mathrm{HC}^{-}$patients. To check separately which tests yielded the overall group difference, separate univariate ANOVAs with surgical treatment as the group factor were performed on the neuropsychological indices. These indicated significantly lower performances of the $\mathrm{HC}^{+}$group in all assessed indices (learning capacity: $F=17.8, \mathrm{p}<0.01$; recognition: $F=4.5, \mathrm{p}=0.045$; rotations: $F=9.8$, $\mathrm{p}=0.04$; failures: $F=12.6, \mathrm{p}=0.001$; spatial visualisation: $F=6.5, \mathrm{p}=0.02$; visuoconstruction: $F=11.6, \mathrm{p}=0.002$ ).

It has to be considered that our visual memory task also poses high constructional demands. To investigate, therefore, to what extent the group differences in memory could be accounted for by the group differences in visuoconstruction, we computed separate ANCOVAs with the visual memory indices as dependent variables including visuoconstruction as a covariate. Group differences in visual memory largely remained, but significances became slightly less impressive (learning capacity: $F=5.4, \mathrm{p}=0.03$, rotations: $F=11.7$, $\mathrm{p}=0.002$, failures: $F=5.5, P=0.03)$. 
POSTOPERATIVE CHANGES

To evaluate the effects of the surgical treatment, ANCOVAs with surgical treatment as the group factor were computed for the pre-post difference scores (preoperative score-postoperative score) incorporating the preoperative scores as covariates. Inclusion of the preoperative values was necessary, as it has often been shown that the preoperative performance level influences the postoperative change. ${ }^{25}$ There were no significant group differences ( $F$ between 0.04 and 2.6 with $\mathrm{p}$ between 0.9 and 0.12 ) thus indicating that the kind of surgical treatment had no different effects on the postoperative development.

To evaluate whether there were any significant postoperative performance changes we computed paired $t$ tests for the total group. These indicated significant postoperative gains for rotations in visual memory $(t=-2.5$, $\mathrm{p}=0.18)$ and for the visuoconstructional abilities $(t=-5.7, \mathrm{p}<0.001)$.

\section{Discussion}

This study assessed right hemispheric cognitive functions in patients with right hippocampal sclerosis who had selective amygdalohippocampectomy $\left(\mathrm{HC}^{+}\right)$and in patients who received right temporal lobe resections which spared the hippocampal tissue $\left(\mathrm{HC}^{-}\right)$. Neuropsychological examinations were done preoperatively and 3 months after surgery.

Preoperative results indicated visual memory deficiencies only for the patients with hippocampal sclerosis. These patients scored significantly worse in the visual memory task than patients with extrahippocampal pathology and their performance was below average compared with healthy controls. The deficits in visual memory could not be attributed to impaired visuoconstructive abilities. The $\mathrm{HC}^{-}$ patients, on the other hand, showed no deficit in any assessed function. The different preoperative performance patterns of $\mathrm{HC}^{+}$and $\mathrm{HC}^{-}$ patients suggest that, within the right temporal lobe, the hippocampus is critically involved in non-verbal memory functions. Hence the results support and extend the findings of studies that have found the same relation in postoperative patients. ${ }^{12-15}$ Visual memory performance could therefore be used in the preoperative neuropsychological examination to determine the functional integrity of the hippocampal structure. An explanation is needed for the total lack of deficiencies in the $\mathrm{HC}^{-}$group. It may be that the applied tests do not assess right temporal cognitive functions beyond the hippocampal functions. Another explanation could be that deficiencies are blurred by the variability of lesion sites in these patients with extrahippocampal lesions.

Regarding postoperative development, the type of surgical treatment had no different effects on the assessed neuropsychological performances. No significant deteriorations were evident for either group, thus supporting the general view that right temporal lobe surgery bears only small risks for additional cognitive deficiencies or exaggerations of preoperative impairments. Significant gains were postopera- tively evident for rotational errors in the visual memory task and for visuoconstructive abilities. Because all patients in our sample were seizure free after surgery, these gains could be interpreted as a release effect of right hemispheric functions beyond the resection site. If we assume a special contribution of the right hippocampus to non-verbal memory, it is rather surprising that excisions of the hippocampus did not exaggerate the preoperative deficiencies, but that the $\mathrm{HC}^{+}$patients even slightly improved in visual memory. Rausch ${ }^{26}$ suggested that the often reported lack of increased deficiencies after a right temporal lobe resection may indicate a comparably large functional plasticity of the right temporal lobe. In this respect, an epileptic hippocampus may be worse than no hippocampus for intrahemispheric or interhemispheric reorganisation.

In conclusion, in line with previous postoperative studies our results verify a special involvement of the right hippocampus in learning and memory for non-verbal material. Patients with hippocampal damage showed preoperatively and postoperatively impaired visual memory performance whereas patients without hippocampal damage exhibited no deficiencies in visual memory. Visual memory deficiencies were not exaggerated after right hippocampal resections. Rather, postoperative data indicated improvements which could be interpreted as release effects and supported the notion that right temporal lobe resections bear only small risks for cognitive deteriorations.

1 Milner B. Psychological aspects of focal epilepsy and its neurosurgical management. In: Purpura DP, Penry JK, Walker RD, eds. Neurosurgical management of the epilepsies. New York: Raven Press, 1975:299-321.

2 Delaney RC, Rosen AJ, Mattson RH, et al. Memory function in focal epilepsy: a comparison of non-surgical, unilateral temporal lobe and frontal lobe samples. Cortex 1980;16:103-17.

3 Ladavas E, Umilta C, Provinciali L. Hemisphere-dependent cognitive performances in epileptic patients. Epilepsia 1979;20:493-502.

4 Majdan A, Sziklas V, Jones-Gotman MJ. Performance of healthy subjects and patients with resection from the anterior temporal lobe on matched tests of verbal and visuoperceptual learning. F Clin Exp Neuropsychol 1996;18:416-30.

5 Giovagnoli AR, Casazza M, Avanzini G. Visual learning on a Giovagnoli AR, Casazza M, Avanzini G. Visual learning on a
selective reminding procedure and delayed recall in patients selective reminding procedure and delayed recall in patien
with temporal lobe epilepsy. Epilepsia 1995;36:704-11.

with temporal lobe epilepsy. Epilepsia 1995;36:704-11.
6 Delaney RC, Prevey ML, Mattson RH. Short term retention with lateralized temporal lobe epilepsy. Cortex 982;22:591-600

7 Ivnik RJ, Sharbrough FW, Laws ER. Effects of anterior temporal lobectomy on cognitive function. F Clin Psychol 1987; 43:128-37.

8 Saykin AJ, Gur RC, Sussman NM, et al. Memory deficits before and after temporal lobectomy: effect of laterality and age of onset. Brain Cogn 1989;9:191-200.

9 Novelly RA, Augustine EA, Mattson RH, et al. Selective memory improvement and impairment in temporal lobectomy for epilepsy. Ann Neurol 1984;15:64-7.

10 Helmstaedter C, Lehnertz K, Grunwald Th, et al. Differential involvement of left temporo-lateral and temporo-mesial structures in verbal declarative learning and memory: evidence from temporal lobe epilepsy. Brain Cogn 1997;35: 110 .

11 Hermann BP, Seidenberg M, Haltiner A, et al. Adequacy of language function and verbal memory performance in unilateral temporal lobe epilepsy. Cortex 1992;28:423-33.

12 Milner B. Visually-guided maze learning in man: effects of bilateral hippocampal, bilateral frontal, and unilateral cerebral lesions. Neuropsychologia 1965;3:317-38.

13 Jones-Gotman M, Milner B. Right temporal-lobe contribution to image-mediated verbal learning. Neuropsychologia 1978;16:61-71.

14 Jones-Gotman M. Memory for designs: the hippocampal contribution. Neuropsychologia 1986;24:193-203.

15 Jones-Gotman M. Right hippocampal excision impairs Jones-Gotman M. Right hippocampal excision impairs
learning and recall of a list of abstract designs. Neuropsychologia 1986;25:659-70

16 Lehrl S. Mehrfachwahl-Wortschatz-Intelligenztest MWT-B. Erlangen: Verlag Dr med Straube, 1978. 
17 Brickenkamp R. Test d2 Aufmerksamkeits-Belastungs-Test. Göttingen: Hogreve, 1978

18 Oldfield RC. The assessment and analysis of handedness: the Edinburgh inventory. Neuropsychologia 1971;9:79.

19 Helmstaedter C, Pohl C, Hufnagel A, et al. Visual learning deficits in non-resected patients with right temporal lobe epilepsy. Cortex 1991;27:547-55

20 Weidlich S, Lamberti G. DCS Diagnostikum für Cerebralschädigung nach F.Hillers. Bern: Huber Verlag, 1980.

21 Helmstaedter C, Pohl C, Elger CE. Eine modifizierte Version des Diagnostikums für Cerebralschäden (DCS) zur Diagnostik räumlich-visueller Gedächtnisdefizite bei Patienten mit Temporallappenepilepsie. In: Scheffner D, ed. Epilepsie 90. Reinbeck: Einhorn-Presse Verlag, 1991:272-9.
22 Tewes U. Hamburg-Wechsler Intelligenztest für Erwachsene Revision 1991. Bern: Verlag Hans Huber, 1991.

23 Horn W. Leistungsprüfsystem L-P-S. Göttingen: Hogrefe, 1983.

24 Lienert GA. Testaufbau und Testanalyse. Weinheim: Verlag Julius Beltz, 1969

25 Chelune GJ, Naugle RI, Lüders H, et al. Prediction of cognitive change as a function of preoperative ability level among temporal lobectomy patients at six months follow up. Neurology 1991;41:399-404.

26 Rausch R. Effects of surgery on cognitive functioning. In: Sackellares JC, Berent S, eds. Psychological disturbances in epilepsy. Boston: Butterworth-Heinemann, 1996:245-58.

\section{NEUROLOGICAL PICTURE}

\section{Subependymal thalamic haemorrhage due to a thalamoperforating artery aneurysm}
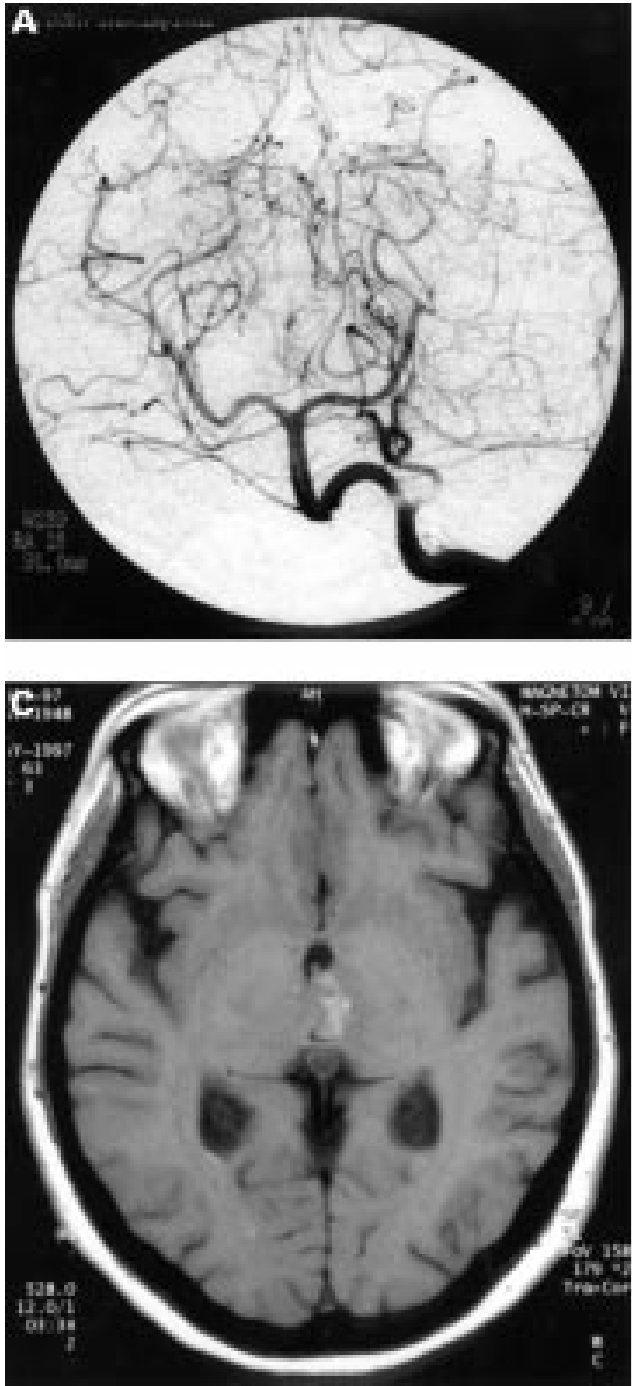

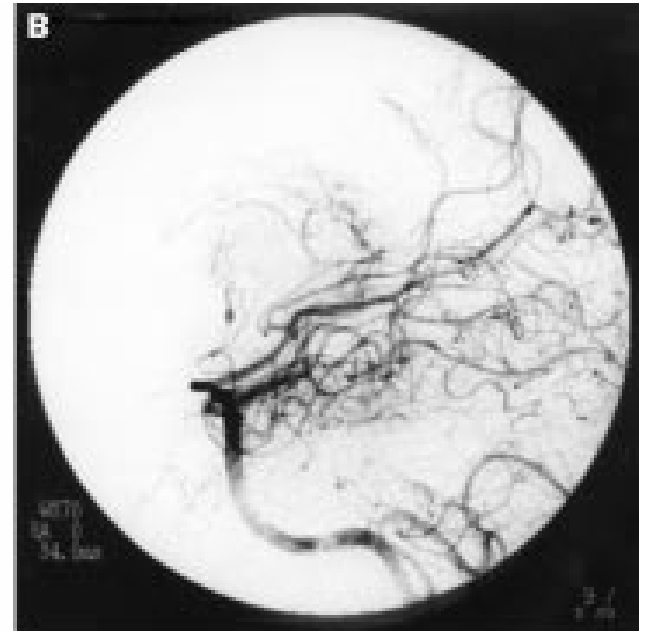

A 49 year old woman was admitted because of sudden onset of severe headache, followed by double vision shortly thereafter. Her medical history was unremarkable. On clinical examination she had mild meningism. She was alert and fully oriented. Arterial blood pressure was $150 / 90 \mathrm{~mm} \mathrm{Hg}$. Neurological findings included a left trochlear nerve palsy, an incomplete Parinaud's syndrome with difficulty on convergence, delayed pupil reaction, and restriction of upward gaze. Brain CT disclosed a small subependymal left thalamic haemorrhage partially occluding the posterior third ventricle. There was minor intraventricular bleeding in the third and fourth ventricles and the posterior horns of the lateral ventricles. Cerebral four vessel digital subtraction angiography showed a left fusiform thalamoperforating artery aneurysm with a 3 $\mathrm{mm}$ diameter (figure, $\mathrm{A}$ and $\mathrm{B}$ ). There were no other abnormalities. An MRI was obtained four days after the bleeding and confirmed the paraventricular location of the haemorrhage (figure C). During her stay in hospital repeated blood pressure measurements were normal. Echocardiography was unremarkable. Erythrocyte sedimentation rate was 30/75. Laboratory studies disclosed a titre of antibodies to 